\title{
Wilms’ Tumor-1 (WT1) Protein in Urinary Exosomes Predicts Risk of Developing Proteinuria in Type-1 Diabetes
}

\author{
Aradhana Mohan', Aditya Upadhyay1, Madan M Godbole ${ }^{1,2}$, Eesh Bhatia² and Swasti Tiwari ${ }^{1 *}$
}

${ }^{1}$ Department of Molecular Medicine \& Biotechnology, Sanjay Gandhi Postgraduate Institute of Medical Sciences, Lucknow, India

${ }^{2}$ Department of Endocrinology, Sanjay Gandhi Postgraduate Institute of Medical Sciences, Lucknow, India

\begin{abstract}
Objective: Previously, we reported presence of Wilms' tumor 1 (WT1) in urinary exosomes in $~ 50 \%$ diabetic (T1D) population without proteinuria as oppose to $100 \%$ in T1D population with proteinuria. Here, we investigated if T1D subjects with detectable WT1 in urinary exosomes (UE) progress to proteinuria faster in a 2 year follow-up study.
\end{abstract}

Method: 52 T1D subjects without proteinuria (age $<60$ years) were enrolled. Urine and blood samples were collected for analysis. WT1 immunoblotting was performed on UE isolated by ultracentifugation of urine samples. Urine microalbumin, serum creatinine and glycated haemoglobin ( $\mathrm{HbA} 1 \mathrm{c})$ were analyzed.

Results: WT1 protein was detected in UE of 28 out of 52 T1D subjects. The data from followed patients, at the end of two years of follow up, revealed that 4 subjects (out of total 19) progressed to proteinuria with urine microalbumin $>20 \mu \mathrm{g} / \mathrm{min}$ in WT1-positive group as oppose to none in WT1 negative group. Moreover, binary logistic regression analysis revealed a significant relation of WT1 presence in UE with increase in urine microalbumin $>5$ $\mu \mathrm{g} / \mathrm{min}$ as indicated by significant Odds ratio of $9.9(95 \% \mathrm{Cl}$ of $92.686, \mathrm{p}=0.04)$. Both groups had statistically similar diabetes duration, age, \% $\mathrm{HbA} 1 \mathrm{c}$ and estimated GFR at the baseline.

Conclusion: Thus, presence of WT-1 in urinary exosomes could potentially predict risk of developing proteinuria in type 1 diabetic patients.

\section{Introduction}

Diabetic nephropathy (DN) is one of the severe complications that affect population with diabetes [1]. The clinical hallmarks of DN includes an initial period of glomerular hyperfiltration, progressive albuminuria, hypertension, followed by a gradual decline in renal function concluding, after 5-15 years, with End-Stage Renal Disease (ESRD) [1-3]. On ESRD, the patients are left with the only choice of renal replacement therapy. This unnecessary pain and economical burden can be avoided by several interventions if the onset and course of development of renal pathology and dysfunction can be predicted at a very early stage of development. Presently, albuminuria is the most recommended screening test for onset and progression of DN. Although its presence is appropriate in patients with advanced diabetic nephropathy but it has limited ability to predict the earliest stages of DN.

This advocates an urgent need for early predictors of microalbuminuria (MA). In this regard, a number of circulating molecules have been proposed as MA predictors such as; homocysteine, aldosterone, B-type natriuretic peptide (BNP), serum mannose binding lectin, osteopontin (OPN) and plasma dipeptidyl peptidase 4 (DPP4) activity [4-7]. In addition, fractional excretion of potassium, and urine immunoglobulin G, Ceruloplasmin, and Transferrin levels were also associated with a risk of developing MA $[8,9]$. Being noninvasive, urine has unique advantages as biomarker source for example Tamm- Horsfall protein (THP), $\alpha-1$ acid glycoprotein, clusterin and progranulin [10], however, it is limited by the presence of abundantnon-specific proteins that could mask potential marker present in trace amount $[1,11]$. This limitation could be overcome by enriching exosomes (UE), nanovesicles released by renal epithelial cells, from total urine samples [12]. UE contain molecules that faithfully reflect the physiological state of their respective cells of origin and thus, appear to serve as a promising tool as biomarker source for renal dysfunction and structural injury [13].
WT1 is known to regulate epithelial-mesenchymal transition process, important step in the pathogenesis and progression of diabetic nephropathy, by transcriptional activation of certain key genes [14]. Furthermore, WT-1 also regulates the expression of the major glomerular structural protein Podocalyxin that serve as early biomarker for DN and provide a molecular basis for the glomerular nephropathy [15]. Recently, we have reported detection of significantly higher level of WT1, a podocyte marker and transcriptional factor, in urinary exosomes of patients with proteinuria and its almost complete absence in non-diabetic healthy controls [16]. Notable, urinary exosomes from all the T1D patients with proteinuria studied were positive for WT1 whereas only $\sim 50 \%$ of the patients without proteinuria had detectable WT1 [16]. Moreover, study by Zhou et al, 2013 has reported the detection of WT1 in animal model of Collapsing Glomerulopathy (CG), Focal Segmental Glomerulosclerosis (FSGS) and Steroid-Sensitive Nephrotic Syndrome (SSNS) patients followed by appearance of proteinuria and glomerular histological damage [17]. Taken together, it can be hypothesized that appearance of WT-1 in urinary exosomes may be a risk of developing proteinuria in diabetic

*Corresponding author: Swasti Tiwari, Associate Professor, Department of Molecular Medicine \& Biotechnology, 4 Floor, PMMSY Building, Sanjay Gandhi Postgraduate Institute of Medical Sciences, Lucknow, India-226014, Tel: 91-522-2495642; Fax: 91 522-2668017; E-mail: tiwari_pgi@yahoo.com, tiwaris@sgpgi.ac.in

Received December 06, 2014; Accepted January 12, 2015; Published January 25, 2015

Citation: Mohan A, Upadhyay A, Godbole MM, Bhatia E, Tiwari S (2015) Wilms Tumor-1 (WT1) Protein in Urinary Exosomes Predicts Risk of Developing Proteinuria in Type-1 Diabetes. J Diabetes Metab 6: 510. doi:10.4172/21556156.1000510

Copyright: $\odot 2015$ Mohan A, et al. This is an open-access article distributed unde the terms of the Creative Commons Attribution License, which permits unrestricted use, distribution, and reproduction in any medium, provided the original author and source are credited. 
Citation: Mohan A, Upadhyay A, Godbole MM, Bhatia E, Tiwari S (2015) Wilms' Tumor-1 (WT1) Protein in Urinary Exosomes Predicts Risk of Developing Proteinuria in Type-1 Diabetes. J Diabetes Metab 6: 510. doi:10.4172/2155-6156.1000510

population. To address this, we planned to follow type-1 diabetic patients with normolabuminuria/ without proteinuria for a period of two years.

52 T1D subjects without proteinuria were randomly enrolled for follow up study. Subjects over 60 years of age were excluded to minimize the effect of age related renal function decline. Spot urine samples, collected at the time of enrollment, were used for enrichment of urinary exosomes as described earlier [16]. All 52 samples of UE were positive for urinary exosomal marker proteins (Tumor susceptibility gene 101(TSG101) and Alix) indicating successful enrichment of UE from urine samples (Figure 1). Moreover, presence or absence of proteinuria does not affect the efficiency of exosomes enrichment as shown in Figure 1A and 1B. However, only 28 subjects (53\%) had detectable levels of WT1 (WT1 positive group) in their UE while in 24 subjects WT1 was undetectable in UE (WT1 negative group). A similar proportion of WT positive diabetic population was found in our earlier study [16]. The patients were followed for a period of two years.

Among the enrolled 52 subjects, urine and blood samples could be collected from only 31 patients at the end of two years. Among them, 19 were from WT1 positive group and 12 from WT1- negative group. Urine microalbumin levels at the end of two years revealed that 4 subjects in WT1 positive group (total 19) progressed to microalbuminuria as oppose to none of the 12 in WT1 negative group (Figure 2, cut-off $>20 \mu \mathrm{g} / \mathrm{min}$ shown by solid reference line). Baseline and follow up values of urine microalbumin of each subject in the two groups has been indicated in Figure 2. Moreover, WT1 positive

A.
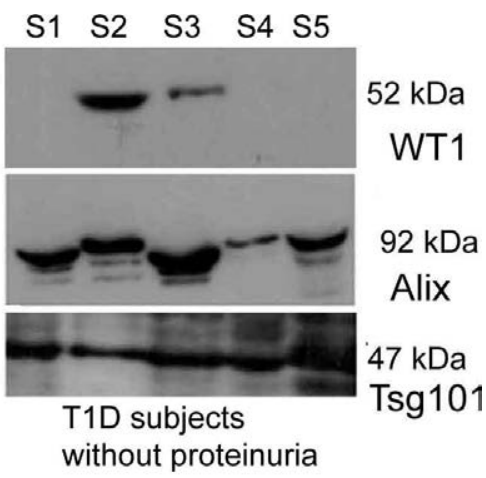

B.
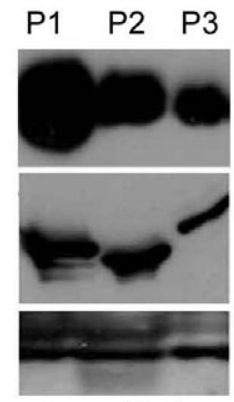

T1D patients with proteinuria

Figure 1: WT1 protein in urinary exosomes of diabetic patients. Representative immunoblots for WT1 in urinary exosome samples from (A) type 1 diabetic subjects (S1-S5) patients without proteinuria at the time of enrollment and (B) type 1 diabetic patients (P1-P3) with proteinuria. All exosomal preparations showed specific band for exosomal marker proteins, Alix and TSG101.

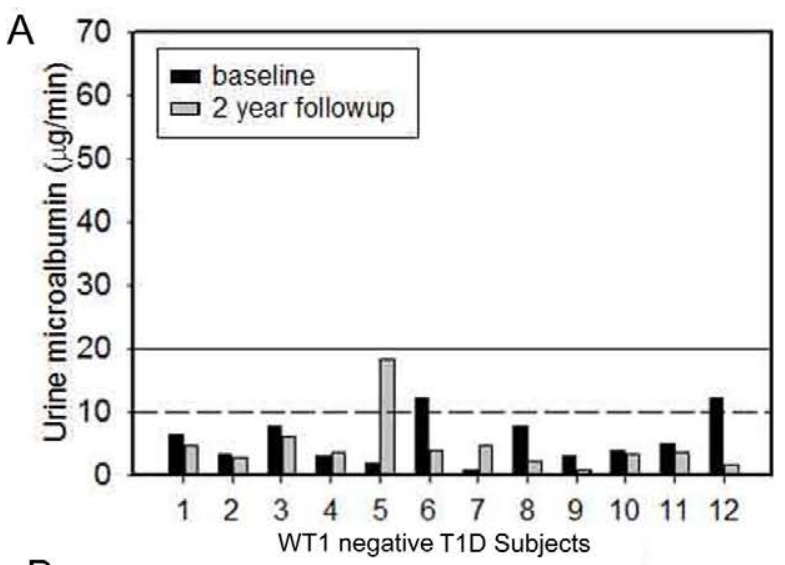

B

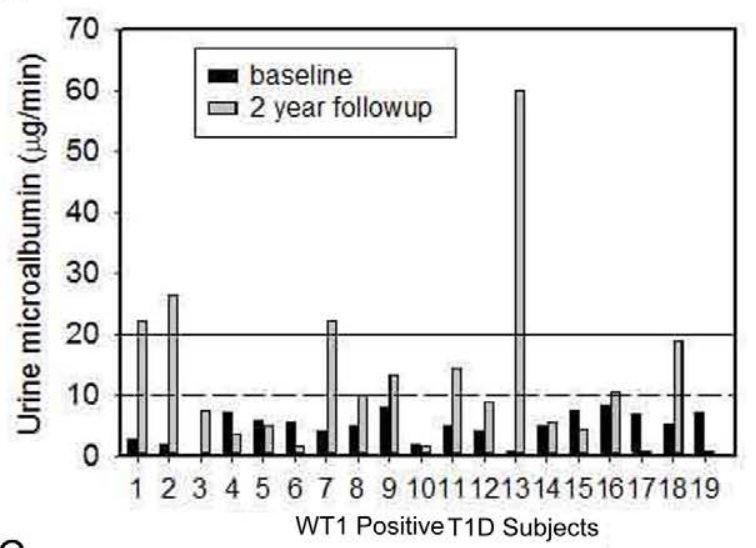

C

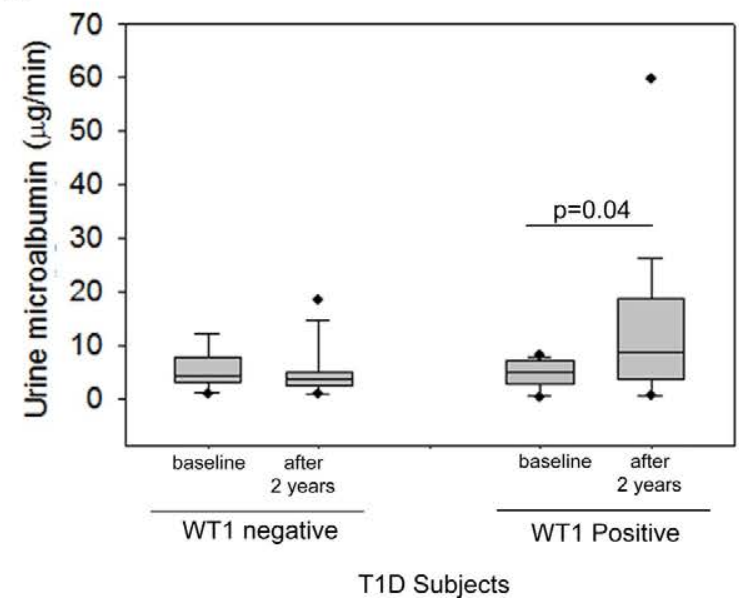

Figure 2: Change in Urine Microalbumin levels in 2 year follow-up. Bar graphs showing urine microalbumin levels at baseline and at the end of 2 year follow-up, in type-1 diabetic subjects (T1D) with (A) undetectable WT1 (WT1 negative), and (B) detectable WT1 (WT1 positive) in urinary exosomes. Below is the box plot $(\mathrm{C})$ comparing the changes in urine microalbumin levels between the two groups. The boxes indicate median and 25th and 75th percentiles; Outliers are indicated by closed dots. $p<0.05$ was considered significant by paired t-test.

population showed significant increase in urine albumin at the end of 2 years of follow-up ( $\mathrm{p}=0.04$, Figure $2 \mathrm{C}$ ).

Notably, degree of albuminuria well below the microalbuminuria cut-off has been reported as a risk factor for complications such as cardiovascular $(\mathrm{CV})$ events in subjects with or without diabetes 
Citation: Mohan A, Upadhyay A, Godbole MM, Bhatia E, Tiwari S (2015) Wilms' Tumor-1 (WT1) Protein in Urinary Exosomes Predicts Risk of Developing Proteinuria in Type-1 Diabetes. J Diabetes Metab 6: 510. doi:10.4172/2155-6156.1000510

Page 3 of 4

\begin{tabular}{|l|c|c|c|}
\hline Indices & WT1 Positive & WT1 Negative & p-value \\
\hline N & 19 & 12 & - \\
\hline Sex (male/female) & $10 / 9$ & $10 / 2$ & $0.2^{*}$ \\
\hline Age (years) & $25 \pm 15$ & $23 \pm 16$ & 0.844 \\
\hline Duration of Diabetes (years) & $11 \pm 9$ & $9 \pm 9$ & 0.438 \\
\hline HbA1c (\%) & $8.2 \pm 1.2$ & $7.3 \pm 1.2$ & 0.068 \\
\hline Serum Creatinine (mg/dl) & $0.85 \pm 0.19$ & $0.77 \pm 0.21$ & 0.371 \\
\hline $\begin{array}{l}\text { Estimated Glomerular } \\
\text { Filteration Rate (eGFR) (ml/ } \\
\text { min/1.73m2) }\end{array}$ & $102 \pm 28$ & $105 \pm 28$ & 0.695 \\
\hline Urine microalbumin (jg/g) & $4.8 \pm 2.5$ & $5.6 \pm 3.7$ & 0.4 \\
\hline
\end{tabular}

All data represented as mean \pm standard deviation of the means. P-values were calculated by Student t-test except for ${ }^{*}$ which was calculated by Fisher exact test.

Table 1: Baseline characteristics of WT1 positive and WT1 negative diabetic subjects.

$[18,19]$. Moreover, urinary albumin excretion $>4.8 \mu \mathrm{g} / \mathrm{min}$ has been reported as a strong and independent determinant of coronary heart disease and death [19]. Therefore, in our study we also analyzed the data using lower cut-offs of urine microalbumin value for proteinuria. With a cut-off urine microalbumin $>10 \mu \mathrm{g} / \mathrm{min}$ (represented by dash line in Figure 2A and 2B), more number of subjects in WT1 positive group progressed to proteinuria (9 out of 19) relative to WT1 negative group (1 out of 12). We used Binary logistic regression analysis to test whether presence or absence of WT1 in urinary exosomes could predict proteinuria (increase in urine microalbumin $>5 \mu \mathrm{g} / \mathrm{min}$ ) in 2 year follow up using the following equation Logit $\mathrm{P}=-0.0953+\left(2.293^{*}\right.$ urine microalbumin). A significant Odds ratio of 9.9 with $95 \%$ CI of 92.686 was found $(\mathrm{p}=0.04)$ suggesting that detection of WT1 in urinary exosomes can predict proteinuria.

Poor glycemic control and diabetic duration could affect the development of proteinuria [20]. This does not appear to be the case in our study since both these parameters; including GFR (estimated Glomerular Filteration Rate eGFR) and urine microalbumin were not significantly different between the two groups at the time of enrollment (Table 1). Although, the baseline HbAlc was trending higher, but not significant ( $\mathrm{p}=0.068)$ in WT1 positive group. Moreover, in both groups, $\mathrm{HbA1c}$ levels did not change significantly in 2 year follow up (Figure 3A). In addition, Linear Regression analysis was performed to determine any relationship between changes in HbAlc levels and urine microalbumin levels in our study (Figure 3B and 3C). Results revealed that baseline $\mathrm{HbAlc}$, and also change in $\mathrm{HbAlc}$ levels had a weak association with urine microalbumin at the end of two year of follow up $(\mathrm{r}=0.02, \mathrm{p}=0.9 ; \mathrm{n}=31)$.

The increased packaging of WT1 in urinary exosomes found in a subset of diabetic subjects may reflect its altered regulation in podocytes and thus could predict the occurrence of proteinuria. This sound reasonable as WT1 has the ability to affect structural as well as the functional integrity of glomerular podocyte, one of the initial steps in the pathogenesis to proteinuria, by regulating the expression of key structural genes [21-24]. WT1 is a transcription factor which belongs to the Cys2-His2 zinc-finger family and reported to regulate the expression of many genes through DNA binding $[15,24,25]$. It plays crucial role in nephrogenesis and podocyte differentiation during embryogenesis [26]. Moreover, nphs1 and podocalyxin, podocyte specific key structural genes were shown to act downstream of WT1 [22]. Thus, we speculate that up-regulation of WT1 in podocytes in response in hyperglycemia-induced-injury in a subset of diabetic population may have resulted in its appearance in urinary exosomes.

In summary, we have shown that among a small cohort of type-

1 diabetic population, with similar duration of diabetes and \% $\mathrm{HbA1c}$, the odds of subjects developing proteinuria (as indicated by increase in urine microalbumin microalbumin $>5 \mu \mathrm{g} / \mathrm{min}$ ) is higher in WT1-positive group in two years compared to WT1 negative group. Thus, presence of WT1 in urinary exosomes could potentially

A

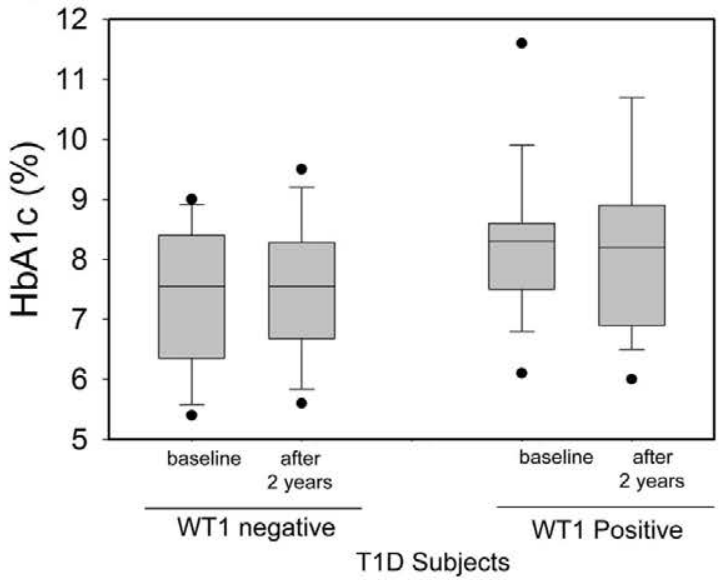

B
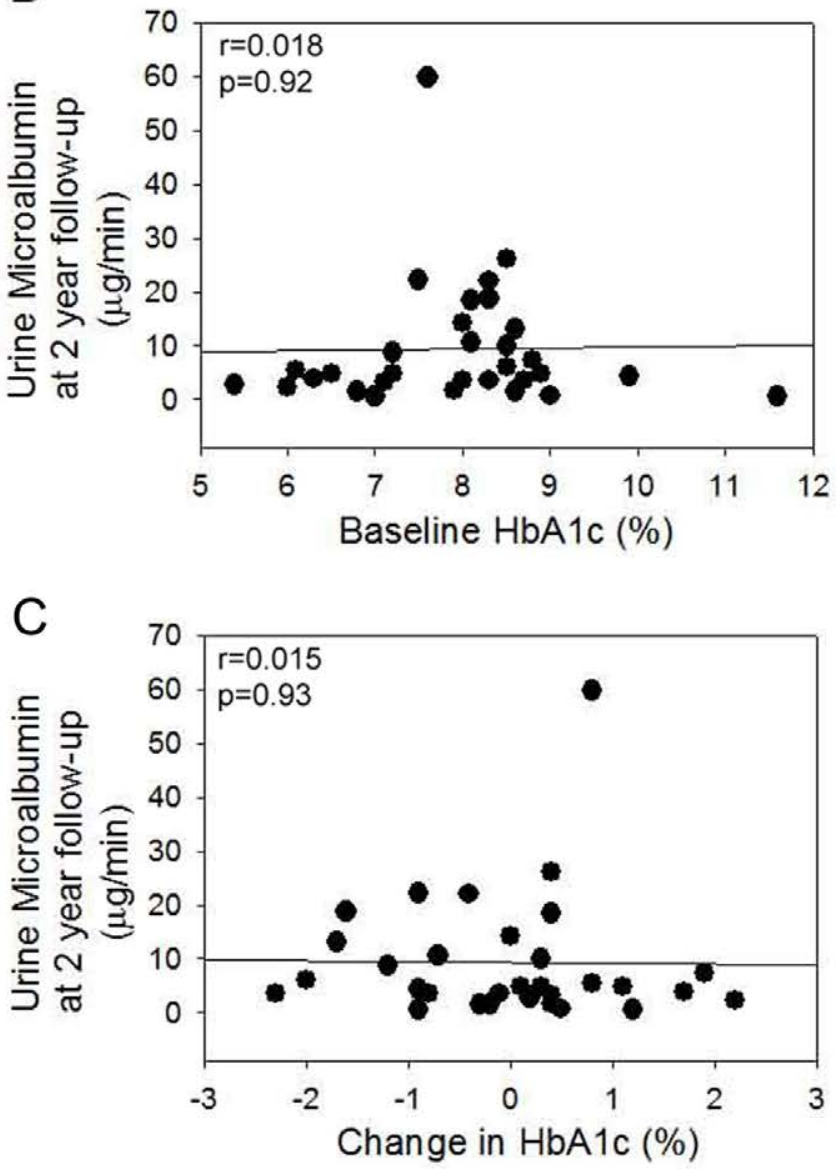

Figure 3: Relationship between $\mathrm{HbA} 1 \mathrm{c}$ and urine microalbumin levels. (A) The box plot shows $\mathrm{HbA} 1 \mathrm{c}$ levels, at baseline and at the end of 2 years of follow-up. The boxes indicate median and 25th and 75th percentiles; Outliers are indicated by closed dots. $\mathrm{HbA} 1 \mathrm{c}$ levels did not change significantly at the end of 2 years. Linear regression analysis showed a weak relationship of urine microalbumin at the end of two year of follow up with (A) baseline $\mathrm{HbA1c}$ and $(B)$ change in HbA1c levels in T1D subjects $(n=31)$. 
Citation: Mohan A, Upadhyay A, Godbole MM, Bhatia E, Tiwari S (2015) Wilms' Tumor-1 (WT1) Protein in Urinary Exosomes Predicts Risk of Developing Proteinuria in Type-1 Diabetes. J Diabetes Metab 6: 510. doi:10.4172/2155-6156.1000510

predict the risk of developing proteinuria in type-1 diabetic subjects. However, further studies, with large cohort and longer follow up, are warranted. Moreover, since $20-30 \%$ of type-1 diabetic patients develop nephropathy, the findings may be helpful in categorizing diabetic subgroup that may go on to develop this complication (Supplementary data).

\section{Acknowledgements}

The authors thank Mr. Praveen Kumar (senior research fellow) for technical support with Ultracentrifugation and, Mr. K. Ramesh Kumar (Department of Endocrinology) and Ms. Shashi Yadav (Department of Molecular Medicine) for their technical assistance with biochemical assays. The authors also thank Mr. Ashish Awasthi (senior research fellow) for assistance with statistical analysis.

\section{Funding}

This work was supported by the Department of Science and Technology and Department of Biotechnology, Government of India and intramural grants to ST. AM was supported by a Senior Research Fellowship from University Grant Commission, India. AU was supported by a Project Senior Research Fellowship from Department of Biotechnology, India.

\section{References}

1. Ben Ameur R, Molina L, Bolvin C, Kifagi C, Jarraya F, et al. (2010) Proteomic approaches for discovering biomarkers of diabetic nephropathy. Nephrology, dialysis, transplantation: official publication of the European Dialysis and Transplant Association - European Renal Association 25: 2866-2875.

2. Marshall SM (2004) Recent advances in diabetic nephropathy. Postgraduate medical journal 80: 624-33.

3. Tesch GH (2010) Review: Serum and urine biomarkers of kidney disease: A pathophysiological perspective. Nephrology (Carlton, Vic) 15: 609-616.

4. Hovind P, Hansen TK, Tarnow L, Thiel S, Steffensen R, et al. (2005) Mannosebinding lectin as a predictor of microalbuminuria in type 1 diabetes: an inception cohort study. Diabetes 54: 1523-1527.

5. Zheng T, Baskota A, Gao Y, Tian H, Yang F (2014) Increased plasma dipeptidy peptidase 4 activities predict new-onset microalbuminuria in association with its proinflammatory effects in Chinese without diabetes: a four-year prospective study. Nephrol Dial Transplant.

6. Gordin D, Forsblom C, Panduru NM, Thomas MC, Bjerre M, et al. (2014) Osteopontin is a strong predictor of incipient diabetic nephropathy, cardiovascular disease, and all-cause mortality in patients with type 1 diabetes. Diabetes care 37: 2593-600.

7. Fox CS, Gona P, Larson MG, Selhub J, Tofler G, et al. (2010) A multi-marker approach to predict incident CKD and microalbuminuria. Journal of the American Society of Nephrology: JASN 21: 2143-2149.

8. Narita T, Hosoba M, Kakei M, Ito S (2006) Increased urinary excretions of immunoglobulin $\mathrm{g}$, ceruloplasmin, and transferrin predict development of microalbuminuria in patients with type 2 diabetes. Diabetes care 29: 142-144.

9. Eghan BA Jr., Frempong MT, Adjei-Poku M (2007) Prevalence and predictors of microalbuminuria in patients with diabetes mellitus: a cross-sectional observational study in Kumasi, Ghana. Ethnicity \& disease 17: 726-730

10. Schlatzer D, Maahs DM, Chance MR, Dazard JE, Li X, et al. (2012) Nove urinary protein biomarkers predicting the development of microalbuminuria and renal function decline in type 1 diabetes. Diabetes care 35: 549-555.

11. Decramer S, Gonzalez de Peredo A, Breuil B, Mischak H, Monsarrat B, et al. (2008) Urine in clinical proteomics. Molecular \& cellular proteomics: MCP 7 : 1850-1862.
12. Pisitkun T, Shen RF, Knepper MA (2004) Identification and proteomic profiling of exosomes in human urine. Proceedings of the National Academy of Sciences of the United States of America 101: 13368-13373.

13. Gonzales PA, Pisitkun T, Hoffert JD, Tchapyjnikov D, Star RA, et al. (2009) Large-scale proteomics and phosphoproteomics of urinary exosomes. Journal of the American Society of Nephrology: JASN 20: 363-79.

14. Miller-Hodges E, Hohenstein P (2012) WT1 in disease: shifting the epithelialmesenchymal balance. The Journal of pathology 226: 229-240.

15. Palmer RE, Kotsianti A, Cadman B, Boyd T, Gerald W, et al. (2001) WT1 regulates the expression of the major glomerular podocyte membrane protein Podocalyxin. Current biology: CB 11: 1805-1809.

16. Kalani A, Mohan A, Godbole MM, Bhatia E, Gupta A, et al. (2013) Wilm's tumor-1 protein levels in urinary exosomes from diabetic patients with or without proteinuria. PloS one 8: e60177.

17. Zhou H, Kajiyama H, Tsuji T, Hu X, Leelahavanichkul A, et al. (2013) Urinary exosomal Wilms' tumor-1 as a potential biomarker for podocyte injury. Am J Physiol Renal Physiol 305: F553-559.

18. Gerstein HC, Mann JF, Yi Q, Zinman B, Dinneen SF, et al. (2001) Albuminuria and risk of cardiovascular events, death, and heart failure in diabetic and nondiabetic individuals. Jama 286: 421-426.

19. Klausen K, Borch-Johnsen K, Feldt-Rasmussen B, Jensen G, Clausen P, et al. (2004) Very low levels of microalbuminuria are associated with increased risk of coronary heart disease and death independently of renal function, hypertension, and diabetes. Circulation 110: 32-35

20. Lee H, Han KH, Lee SE, Kim SH, Kang HG, et al. (2012) Urinary exosoma WT1 in childhood nephrotic syndrome. Pediatric nephrology (Berlin, Germany) 27: $317-320$

21. Zhang A, Huang S (2012) Progress in pathogenesis of proteinuria. International journal of nephrology 2012: 314251.

22. Guo JK, Menke AL, Gubler MC, Clarke AR, Harrison D, et al. (2002) WT1 is a key regulator of podocyte function: reduced expression levels cause crescentic glomerulonephritis and mesangial sclerosis. Human molecular genetics 11: 651-659.

23. Wagner N, Wagner KD, Xing Y, Scholz H, Schedl A (2004) The major podocyte protein nephrin is transcriptionally activated by the Wilms' tumor suppressor WT1. Journal of the American Society of Nephrology: JASN 15: 3044-3051.

24. Hosono S, Luo X, Hyink DP, Schnapp LM, Wilson PD, et al. (1999) WT1 expression induces features of renal epithelial differentiation in mesenchyma fibroblasts. Oncogene 18: 417-27.

25. Zhou H, Cheruvanky A, Hu X, Matsumoto T, Hiramatsu N, et al. (2008) Urinary exosomal transcription factors, a new class of biomarkers for renal disease. Kidney international 74: 613-621.

26. Su J, Li SJ, Chen ZH, Zeng CH, Zhou H, et al. (2010) Evaluation of podocyte lesion in patients with diabetic nephropathy: Wilms' tumor-1 protein used as a podocyte marker. Diabetes research and clinical practice 87 : 167-175. 